\title{
Analysis and Prospect of Targeted Therapy for Head and Neck Tumors
}

\author{
Huang Yizhou \\ Bengbu Medical College, Bengbu 233003, China \\ Email: 321230399@qq.com
}

Keywords: Head and neck tumor, targeted therapy, inhibitor review

\begin{abstract}
Head and neck tumors account for 30\% of malignant tumors in the body, which is a key topic in the world. The comparative data of radiotherapy, surgical therapy or chemotherapy for the treatment of these diseases are given. The treatment of epidermal growth factor receptor inhibitor, cyclin dependent kinase inhibitor and insulin-like growth factor receptor inhibitor were analyzed. It is pointed out that molecular targeted therapy is a targeted blocker to interfere with the signal transduction pathway, which is regulated by the specific molecule and is closely related to the occurrence of tumor, so as to inhibit tumor growth and metastasis.
\end{abstract}

\section{Introduction}

It has been found that malignant tumors are more dangerous and difficult to cure. As far as tumor types are concerned, head and neck tumors are the fifth most common malignant tumor in the world [1].The occurrence frequency of this tumor is relatively high in China, and the most common type of tumor is head and neck squamous cell carcinoma. Clinically, squamous cell carcinoma of the head and neck refers to squamous cell carcinoma that occurs in the lips, hypopharynx, oropharynx and oral cavity, with a high mortality rate of[1-3].Early patients with head and neck squamous cell carcinoma usually get good results after surgical treatment, but because of the lack of understanding of this type of tumor, most patients come to visit, their condition has developed to the local late stage[2].The related literature showed that the majority of patients with head and neck tumors were advanced type and local advanced tumors, and the proportion of them was between $70 \%$ and $80 \%$.These diseases are generally treated with radiotherapy, chemotherapy and surgery. Radiotherapy and chemotherapy have great limitations, one is the patient's healthy cell also have harmful and toxic side effects. Second, the long-term survival rate of patients with advanced tumors was increased by about $20 \%$, and the effect of treatment was not good [3].Third, during the course of radiotherapy and chemotherapy, the patients' salivary glands in their inability to eat smoothly or completely, leading to the gradual deterioration of the patient's body, such as malnutrition, poor resistance, and so on. In contrast to radiotherapy and chemotherapy, the application of targeted therapy brings a break through to the treatment of patients [4].In the course of actual tumor 
targeting therapy, the use of its drugs is based on its mechanism of action, and the use of drugs is based on the characteristics of the disease [5-6].Such as: cetuximab, erlotinib and bevacizumab, etc. Traditional therapy is radiotherapy or chemotherapy. The most typical problem in patients after treatment is local recurrence after treatment [7-8].Local recurrence is an important cause of death in the head and neck cancer. More than 50\% of the patients with head and neck cancer have only local recurrence and nearly $90 \%$ of the distant metastases have local lesions.50\% of the local recurrence patients have poor prognosis, the median survival time is only 6 9 months, the one year survival rate is less than $33 \%$,and the recurrence or metastasis patients have more prognosis, The median survival time was only 3 4 months, and the 1-year survival rate was even less than 5\%[9-11].For recurrent patients, the median total survival time of recurrent patients with cisplatin/platinum combined with paclitaxel was about 3 months. Because of the importance of preservation of anatomical structure and organ function, the treatment of head and neck cancer is very complicated [12].

\section{Analysis of targeted therapy for head and neck tumors}

\subsection{Treatment of Epidermal Growth Factor Receptor Inhibitor}

For the treatment of head and neck neoplasms, it is mainly through drug inhibition treatment [1314].Such as Epidermal Growth Factor Receptor Inhibitors ( EGFRI ).EGFRI has specific characteristics, which can effectively bind Epidermal Growth Factor Receptor ( EGFR ) with Extracellular Kinase ( EK ), thus effectively induce tumor cells to apoptosis[15].It is analyzed that the inhibitor can effectively treat head and neck tumors. The main factors were as follow:

(1) The inhibitor had resistance to tumor cells, and apoptosis of tumor cells was induced under the condition of induction [16-17].

(2) Inhibitors have inhibitory effect on blood vessels: tumor has the characteristic of diffusivity, and its main expansion medium is blood vessel [18-19].The main mechanism of the inhibitor is to inhibit tumor angiogenesis, to block the access of tumor cells to nutrient supply, and to regulate the activity of proteolytic enzyme and the expression of vascular endothelial growth factor on tumor cells. And then multiple targets play a good role in anti-tumor angiogenesis [20].Not only can the patient's neovascularization inhibition, but also effectively reduce the number of tumor microvessels. At the same time, the tumor was controlled [21-22].

(3) It can interfere with the metastasis of tumor cells [23].

(4) Toxic and side effects to tumor cells: this treatment method can stabilize the blood vessels temporarily by inhibiting the function of endothelial cells and tumor cells together, and then effectively inhibiting tumor cells. The window of normalization of blood vessels appears [24]. Koutcher et al.[25]retrospectively analyzed the efficacy of two different treatment patterns in 2011.125 of 174 newly diagnosed head and neck squamous cell carcinoma patients received cisplatin based radiotherapy and chemotherapy.49 patients received EGFRI combined with radiotherapy. The two-year local recurrence rates were $5.7 \%$ and $39.9 \%$ respectively. And failure free survival rates were $87.4 \%$ and $44.5 \%$,respectively. The overall survival rate was $92.8 \%$ and $66.6 \%$,respectively.It can be seen that radiotherapy combined with epidermal growth factor receptor inhibitor can improve the local recurrence rate and overall survival rate, showing some advantages.

\subsection{Analysis of Cyclin-dependent Kinase Inhibitors}

Cyclin-dependent kinases ( CDKs ) are the key proteins to regulate the progress of cell cycle. When CDKs are abnormal in the process of regulating cell cycle, they will lead to the occurrence of 
tumor. Cyclin dependent kinase inhibitors can inhibit the proliferation of tumor cells at the cell cycle level by inhibiting CDKs. As early as 1998, Patel et at found that the cyclin dependent kinase inhibitor, Flapentine, had antitumor activity on HNSCC cells. At present, cyclin dependent kinase inhibitors, including SNS032 and 7-hydroxystaurosporine (UCN01), are still under study[26-27],but mainly focus on hematological tumors, and the research on head and neck squamous cell carcinoma needs to be further elucidated.

\subsection{Treatment of Insulin-like Growth Factor Receptor Inhibitors}

The receptor family of Insulin-like Growth Factoris commonly found in higher eukaryotes and is involved in the growth and development of histocytes[28].Studies have shown that the mechanism that plays an important role in the development of many malignant tumors is to activate many signal transduction pathways, such as MAPK, AKT, and so on, thus triggering signal pathway such as protein synthesis, anti-apoptosis and proliferation [29].It is believed that IGF can promote mitosis, and its expression level is positively correlated with cancer risk, tumor malignancy and disease progression[30].The result showed that the expression of IGF in head and neck squamous cell carcinoma was significantly increased, and the enhanced IGF signal affected the tumorigenicity, proliferation and motility of human head and neck squamous cell carcinoma cell lines, and even had the potential to induce the second primary tumor [31].However the positive correlation between the expression of IGF and the prognosis of head and neck squamous cell carcinoma has not been confirmed, although there are a variety of IGF targeted drugs, such as Figitumumab, which is the most deeply studied IGF antagonist. It has been studied in many kinds of malignant tumors, but it has not got ideal results in a series of combined use. The treatment of IGF targeted drugs in head and neck squamous cell carcinoma needs further study.

\subsection{Gene Therapy for head and neck tumors}

The purpose of gene therapy in the treatment of tumor disease is mainly through correction and compensation [32-33].The so-called gene therapy is to induce exogenous functional genes into the cells of the patients, correct the congenital metabolic abnormalities of the patients, compensate for the deletion of the genes and introduce new functions into the cells [34-45].To achieve the goal of healing through the above mentioned action.

\section{Conclusion}

Nowadays, the key problem is to solve the differentiation factor of inhibitors in tumor cell. If further research is carried out effectively, we can provide new targets for treatment [36].At present, targeted therapy, such as EGFRI, has been shown to be effective in the treatment of head and neck neoplasms. It has the characteristic of specificity and has the function of blocking, thus effectively inducing the apoptosis of tumor cells in patients. Gene therapy induced exogenous functional genes into the cells of the patients, corrected the congenital metabolic abnormalities of the patients, compensated for the deletion of the genes and introduced new functions into the cells. All belong to target therapy [37].However, there are still some limitations in the treatment of recurrent and metastatic tumors. Therefore, the stability of the disease should be taken as the basis in the treatment of the disease. At the same time, in the targeted treatment of head and neck tumors, in addition to effective treatment, attention should be paid to dietary nursing intervention. During the treatment period, patients with malignant tumors of head and neck are prone to malnutrition, but it can be said that many reasons can lead to malnutrition, not only the treatment and disease itself, but also the influence of patients' own factors[38-39].The proportion of protein and food calories 
should be supplement with water soluble vitamins and trace elements electrolytes and amino acids once the patients have serious adverse effects of radiotherapy. At the same time, we should pay attention to nursing the patients with oral mucositis, and encourage the patients to drink water above $2500 \mathrm{ml}$ every day in order to moisten the oral mucosa and promote the effect of toxic substances excretion[40].After eating daily, use a soft toothbrush and fluorine toothpaste to clean the mouth to avoid ulcers.

\section{References}

[1] Corey J. Langer MD.Targeted therapy in head and neck cancer †[J]. Tumor Biology, 2012, 33(3):707.

[2] Ma Yan,Sun Lei,Yu Lei,et al.Advances in targeted treatment of head and neck neoplasms [J].Chinese Journal of Cancer Biotherapy,2014, 21(3):342-347.

[3] Singer S, Arraras J I, Baumann I, et al. Quality of life in patients with head and neck cancer receiving targeted or multimodal therapy - Update of the EORTC QLQ-H\&N35, Phase I[J]. Head \& Neck, 2013, 35(9):1 331.

[4] Sacco A G, Worden F P. Molecularly targeted therapy for the treatment of head and neck cancer: a review of the ErbB family inhibitors:[J]. Onco Targets Ther, 2016, 9:1 927-1 943.

[5] Gao Yang. Progress in Molecular targeted Therapy of head and neck malignant tumors [J]. Comprehensive clinical practice in China, 2010, 26(5):549-551.

[6] Du Y, Peyser N D, Grandis J R. Integration of molecular targeted therapy with radiation in head and neck cancer.[J]. Pharmacology \& Therapeutics, 2014, 142(1):88-98.

[7] Chang J W, Kim C H. Molecular Targeted Therapy of Head and Neck Cancer: Promising Molecular Targets of PostEpidermal Growth Factor Receptor Era[J]. Korean Journal of Otorhinolaryngology-Head and Neck Surgery, 2014, 57(9):575.

[8] ShangDandan,Chen Peng. Advances in targeted treatment of head and neck squamous cell carcinoma [J].Chinese Journal of Geriatrics Stomatology,2016, 14(2):120-124.

[9] Seiwert TY,Salama JK,Vokes EE.The chemoradiation paradigm in head and neck cancer[J].Nat Clin Pract Oncol,2007,4(3):156-171

[10] Argiris A,Li Y,Forastiere A.Prognostic factors and long term survivorship in patients with recurrent or metastatic carcinoma of the head and neck,an analysis of 2 Eastern Cooperative Oncology Group randomized trials[J].Cancer,2004,101(10):2 222-2 229

[11] Leon X,Hitt R,Constenla M,et al.A retrospective analysis of the outcome of patients with recurrent and/or metastatic squamous cell carcinoma of the head and neck refractory to a platinum-based chemotherapy [J].Clin Oncol (R Coll Radiol),2005,17(6):418-424

[12] Moreira J, Tobias A, O'Brien M P, et al. Targeted Therapy in Head and Neck Cancer: An Update on Current Clinical Developments in Epidermal Growth Factor Receptor-Targeted Therapy and Immunotherapies. [J]. Drugs, 2017, 77(8):1-15.

[13] Kerk S, Finkel K, Pearson A T, et al. 5T4-targeted therapy ablates cancer stem cells and prevents recurrence of head and neck squamous cell carcinoma[J]. Clinical Cancer Research An Official Journal of the American Association for Cancer Research, 2016.

[14] Zhang Xu. Treatment of head and neck neoplasms [J]. medicine 2016(11):00157-00158.

[15] Fukuda S, Hatakeyama H. Current Research into Head and Neck Cancer Molecular Targeted Therapy and Human Papilloma Virus Infection in Head and Neck Squamous Cell Carcinoma[J]. Practica oto-rhino-laryngologica, 2012, 105(3):183-191.

[16] Byers L A, Diao L, Ng P K S, et al. Proteomic profiling of HPV-positive head and neck cancer to identify new candidates for targeted therapy.[J]. Journal of Clinical Oncology, 2014.

[17] Jiang Huiyuan, Jiang Bin, Liu Feng, et al. Expression and clinical significance of PD-L1 in head and neck tumors [J]. Journal of Clinical Oncology, 2017, 22(2):124-127.

[18] Wang L X, Agulnik M. Promising new molecular targeted therapies in head and neck cancer.[J]. Drugs, 2013, 73(4):315-325.

[19] Marcu L G, Marcu D. The effect of targeted therapy on recruited cancer stem cells in a head and neck carcinoma model[J]. Cell Proliferation, 2017,19 (23):1789.

[20] Christy A W, Bojan A. Targeted therapy: A novel approach in head and neck cancer.[J]. Indian Journal of Dental Research,2013, 24(2):261.

[21] Guo Wei. Advances in targeted treatment of advanced oral and maxillofacial squamous cell carcinoma [J]. Journal of Oral and Maxillofacial surgery, 2012, 22(2):77-81.

[22] Xiaojing J. Research of targeted therapy in head and neck cancer[J]. Chinese Journal of Cancer Biotherapy, 2014, 
21(3):342-347.

[23] Worsham M J, Ali H, Dragovic J, et al. Molecular characterization of head and neck cancer: how close to personalized targeted therapy?[J]. Molecular Diagnosis \& Therapy, 2012, 16(4):209-210.

[24] Miao Xiangwan, Xie Minqiang. Advances in molecular targeted therapy of head and neck tumors [J]. Chinese Journal of Otolaryngology and skull Base surgery, 2017, 23(3):290-294.

[25] Koutcher L,Sherman E,Fury M,et al.Concurrent cisplatin and radiation versus cetuximab and radiation for locally advanced head and neck cancer [J].Int J Radiat Oncol Biol Phys,2011,81(4):915-922

[26] Meng H, Jin Y, Liu H, et al. SNS032 inhibits mTO RC1/mTO RC2 acti-vity in acute myeloid leukemia cells and has syner gistic activity with perifosine against Akt [J] . J Hematol Oncol, 2013, 6: 1831.

[27] Gojo I, Perl A, Luger S, et al. Phase I study of UCN01 and perifosine in patients with relapsed and refractory acute leukemias and highrisk myelodysplastic syndrome [J] . Invest New Drugs, 2013, 31( 5) : $1217-1227$.

[27] Haisa $M$, The type 1 insulin- like growth factor receptor signalling system and targetedtyrosine kinase inhibition in cancer[J]. J Int Med Res, 2013, 41(2): 253-264.

[29] Shin DH, Min HY, El-Naggar AK, et al. Akt/mTOR counteract the antitumor activities of cixutumumab, an anti- insulin- like growth factor I receptor monoclonal an tibody[J]. Mol Cancer Ther , 2011, 10(12): 2 437- 2 448.

[30] Limesand KH, Chibly AM, Fribley A. Impact of targeting insulin- like growth factor signaling in head and neck cancers [J]. Growth Horm IGF Res, 2013, 23(5): 135-140.

[31] Jameson MJ, Beckler AD, Taniguchi LE, et al. Activation of the insulin- like growth factor- 1 receptor induces resistance to epidermal growth factor receptor antago- nism in head and neck squamous carcinoma cells [J]. Mol Cancer Ther, 2011, 10(11): 2 124-2 134.

[32] Rieke DT, Klinghammer K, Keilholz U. Targeted Therapy of Head and Neck Cancer.[J]. Oncology research and treatment, 2016, 39(12):7803

[33] Sun Lili, Bai Yuxian. Advances in targeted treatment of head and neck squamous cell carcinoma [J]. Chinese Journal of clinicians: electronic Edition,2013, 7(22):10271-10274.

[34] Nakashima T. Molecular targeted therapy for head and neck cancer[J]. Nippon Koku Geka Gakkai Zasshi, 2014, 60(7):404-406.

[35] Strieth S, Ernst B P. [Recent Developments Towards Molecularly Targeted Therapy of Head and Neck Cancer].[J]. Laryngo- rhino- otologie, 2017,22 (21):280-283.

[36] Zhou Lian, Luo Wenlong. Advances in targeted treatment of laryngeal malignant tumors [J]. Journal of Applied Medicine, 2014(13):2168-2171.

[37] Du Lifa, Wang Junjie. Basic study of epidermal growth factor receptor targeted therapy for head and neck squamous cell carcinoma [J]. Cancer Progress,2012, 10(4):355-359.

[38] Xu Haixia, Peng Shi Dong. Research progress of BRMS1 gene in head and neck cancer [J]. Inner Mongolia Journal of medicine, 2015, 47(9):1 063-1 066.

[39] Shao Xiaolin, Zhang Tao. Advances in the study of long chain non-coding RNA associated with head and neck neoplasms [J]. Journal of Applied Oncology, 2016, 30(1):88-92.

[40] Byeon H K. Crosstalk Mechanisms Following Targeted Therapy in Head and Neck Cancer[J]. 2016, 59(3):181 